\title{
On the strong difference in reactivity of acyclic and cyclic diazodiketones with thioketones: experimental results and quantum-chemical interpretation
}

\author{
Andrey S. Mereshchenko ${ }^{1}$, Alexey V. Ivanov ${ }^{1}$, Viktor I. Baranovskii ${ }^{1}$, Grzegorz Mloston², \\ Ludmila L. Rodina ${ }^{1}$ and Valerij A. Nikolaev*1
}

\section{Full Research Paper}

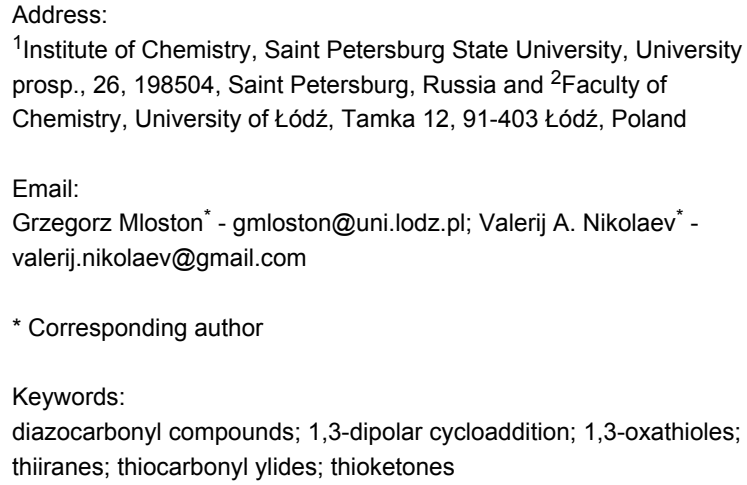

Beilstein J. Org. Chem. 2015, 11, 504-513. doi:10.3762/bjoc.11.57

Received: 02 January 2015

Accepted: 27 March 2015

Published: 20 April 2015
Dedicated with all kind wishes to Honorary Professor of Saint-Petersburg State University, Professor Rolf Huisgen (Munich) on the occasion of his 95th birthday.

(C) 2015 Mereshchenko et al; licensee Beilstein-Institut. License and terms: see end of document.

\begin{abstract}
The 1,3-dipolar cycloaddition of acyclic 2-diazo-1,3-dicarbonyl compounds (DDC) and thioketones preferably occurs with $Z, E$ conformers and leads to the formation of transient thiocarbonyl ylides in two stages. The thermodynamically favorable further transformation of $\mathrm{C}=\mathrm{S}$ ylides bearing at least one acyl group is identified as the 1,5-electrocyclization into 1,3-oxathioles. However, in the case of diazomalonates, the dominating process is 1,3-cyclization into thiiranes followed by their spontaneous desulfurization yielding the corresponding alkenes. Finally, carbocyclic diazodiketones are much less reactive under similar conditions due to the locked cyclic structure and are unfavorable for the 1,3-dipolar cycloaddition due to the Z,Z-conformation of the diazo molecule. This structure results in high, positive values of the Gibbs free energy change for the first stage of the cycloaddition process.
\end{abstract}

\section{Introduction}

Dipolar cycloadditions of diazo compounds have been of great interest for a long time as they provide a means for the preparation of a wide variety of nitrogen containing heterocyclic compounds [1-5]. These reactions are generally known for diazoalkanes and 2-diazocarbonyl compounds, whereas similar processes with 2-diazo-1,3-dicarbonyl compounds (DDC) are far less common [6-10], and reported literature data on this matter are somewhat contradictory. For example, it was established that diazomalonate and diazodimedone do not react under standard conditions with the $\mathrm{C}=\mathrm{S}$ bond of thiobenzophenone $[11,12]$, which is one of the most reactive "superdipolarophiles" known [13]. On the other hand, it was recently demonstrated 
that diazoacetylacetone, even at room temperature, easily reacts with $\mathrm{Ph}_{2} \mathrm{C}=\mathrm{S}$, giving rise to the formation of 1,3-oxathiole derivatives $[14,15]$, which are considered to be the typical final products of the 1,3-dipolar cycloaddition of diazoketones to the $\mathrm{C}=\mathrm{S}$ bond $[16-18]$.

In this regard, we performed a detailed experimental study of reactions of a series of 2-diazo-1,3-dicarbonyl compounds 1 with aromatic and aliphatic thioketones $\mathbf{2}$, and it was established that they occurred in a varied manner: acyclic diazodicarbonyl compounds 1 readily reacted with thioketones $\mathbf{2}$, whereas carbocyclic diazodiketones were essentially indifferent to aromatic and aliphatic thioketones under similar reaction conditions (Scheme 1) [14,15,19-21].

Generally, the main reaction products formed in these reactions were 1,3-oxathioles $\mathbf{3}$. However, in some cases, thiiranes $\mathbf{4}$ and alkenes $\mathbf{5}$ were isolated from the reaction mixture as well $[19,20]$ (Scheme 1). In addition, it was established that in the reaction of diazomalonate with aliphatic 2,2,4,4-tetramethyl-3thioxocyclobutanone, the ratio of the reaction products $\mathbf{3}$ and 5 strongly depends on the reaction conditions: at room temperature, a mixture of 1,3-oxathiole 3 and alkene 5 (4:1) was formed, whereas at $80{ }^{\circ} \mathrm{C}$, product $\mathbf{5}$ was formed exclusively (81\%) [20].

In order to explain the obtained results and to elucidate the reaction pathway (which governs reactions of DDC 1 with aromatic and aliphatic thioketones 2), detailed quantum-chemical calculations of the relative energy of the reagents, reaction intermediates, transition states, and reaction products on the potential energy surfaces were carried out. The results of the performed study are summarized and discussed in the present publication.

\section{Results and Discussion}

The subjects of the quantum-chemical calculations were the same diazodicarbonyl compounds and thioketones that were applied in the earlier experimental studies, namely: acyclic diazodiketones 1a,b, diazoketoester $\mathbf{1 c}$, diazomalonate 1d, and carbocyclic diazodiketones $\mathbf{1 e}, \mathbf{f}$, along with thiobenzophenone (2a) and 2,2,4,4-tetramethyl-3-thioxocyclobutanone (2b) as dipolarophiles (Figure 1).

\section{2-diazo-1,2-dicarbonyl compounds}<smiles>[R]C(=O)C(=N)C(=O)O[13CH3]</smiles><smiles>S=C(c1ccccc1)c1ccccc1</smiles>

2a

$\mathrm{R}, \mathrm{R}^{1}=\mathrm{Me}, \mathrm{Me}(\mathrm{a}) ; \mathrm{Ph}, \mathrm{Ph}(\mathrm{b})$ $\mathrm{Me}, \mathrm{OMe}(\mathrm{c}) ; \mathrm{OMe}, \mathrm{OMe}(\mathrm{d})$

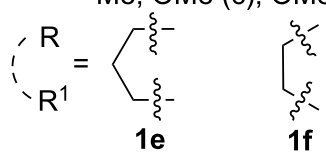<smiles>CC1(C)C(=O)C(C)(C)C1=S</smiles>

2b

Figure 1: Diazo compounds 1 and thioketones 2 used in the study.

The calculations were performed at the density functional theory (DFT) level using the 6-31G(d) basis set. For reactions of diazo compounds 1a-f with thiobenzophenone (2a), the solvent was simulated using the polarizable continuum model

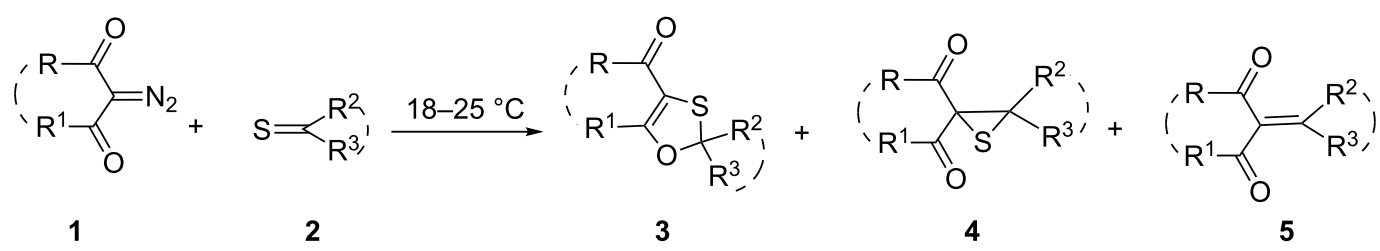

\begin{tabular}{ccccc}
\hline \multirow{2}{*}{ Diazo compounds } & $\mathrm{R}--\mathrm{R}^{1}$ & \multicolumn{3}{c}{ Reaction product yields, \% } \\
\cline { 3 - 5 } & $\mathrm{3e}, \mathrm{3e}$ & $81^{\mathrm{a}} ; 88^{\mathrm{b}}$ & $\mathbf{4}$ & $\mathbf{5}$ \\
\hline \multirow{2}{*}{ Acyclic } & $\mathrm{Mh}, \mathrm{Ph}$ & $40^{\mathrm{a}} ; 44^{\mathrm{b}}$ & & $6^{\mathrm{b}}$ \\
\hline diazodiketones & $\mathrm{Me}, \mathrm{OMe}$ & $45^{\mathrm{a}} ; 77^{\mathrm{b}}$ & - & - \\
\hline \multirow{2}{*}{ Diazoesters } & $\mathrm{OMe}, \mathrm{OMe}$ & $65^{\mathrm{b}}$ & $32^{\mathrm{a}}$ & $13^{\mathrm{b}}$ \\
\hline Carbocyclic & $\left(\mathrm{CH}_{2}\right)_{3}$ & \multirow{2}{*}{ Do not react at similar conditions } \\
diazodiketones & $\left(\mathrm{CH}_{2}\right)_{2}$ & & & \\
\hline
\end{tabular}

aWith aromatic thiobenzophenone. ${ }^{b}$ With aliphatic thioxocyclobutanone. 
(PCM) [22], and for the case of thioketone $\mathbf{2 b}$, all calculations were performed for the gas phase.

\section{Reactions with thiobenzophenone (2a)}

By comparison with the literature data [16-18], one can assume that the multistep reactions of DDC $\mathbf{1 a - d}$ and thione $\mathbf{2 a}$ are initiated by 1,3-cycloaddition of the diazogroup with the $\mathrm{C}=\mathrm{S}$ bond (step 1), followed by decomposition of thiadiazoline 6 formed (step 2) and competitive electrocyclization of the intermediate thiocarbonyl ylide 7 either into 1,3-oxathiole 3 or thiirane 4 (step 3) (Scheme 2).

According to the latest molecular orbital (MO) theory, reactions of diazodicarbonyl compounds with a dipolarophile are cycloaddition processes of type II (HOMO, LUMO controlled) [23-25]. In order to confirm the proposed reaction mechanism and to explain the experimental results, geometries of the stationary points (i.e., reagents, products, intermediates, and the appropriate transition states) on the potential energy surface of the reaction of the diazo compounds $\mathbf{1 a}-\mathbf{f}$ with thiobenzophenone (2a) were located. The optimized structures of reagents, intermediates, and products were found to be characterized by the absence of the imaginary part of the frequency, and transition states contained only one imaginary frequency component. The calculated intrinsic reaction coordinate (IRC) paths demonstrated that the stationary points are effectively connected to each other.

It was found that the $E, Z$-conformations of acyclic DDC 1a-d have the lowest energy as compared to their $Z, E-, Z, Z$ - and $E, E$ counterparts [26-28] (up to $4.7-5.0 \mathrm{kcal} / \mathrm{mol}$ ). Because of this, calculations were carried out for the $E, Z$-conformers of acyclic 1a-d and the $Z, Z$-locked conformation of carbocyclic DDC 1e,f. The optimized structures of the $E, Z$-conformers for diazo compounds 1a-d are shown in Figure 2.

The structural formulas and the corresponding, optimized geometry energy for the first and the second reaction steps (cycloaddition of $\mathbf{1}$ with 2a and decomposition of 1,3,4-thiadiazolines 6) are given in Table 1. The positive values of the Gibbs free energy change for the first stage of the process (Table 1; $\Delta G_{1}$ ) demonstrate that formation of thiadiazolines $\mathbf{6}$ from $\mathbf{1 a - d}$ and $\mathbf{2 a}$ is thermodynamically unfavorable. However, the total value of the Gibbs free energy change for the formation of molecular nitrogen and thiocarbonyl ylide 7 from 1a-d and 2a, $\Delta G_{1-7}=\Delta G_{1}+\Delta G_{2}$, is negative.

Therefore, the formation of the intermediate thiocarbonyl ylides of type $\mathbf{7}$ from $\mathbf{1} \mathbf{a}-\mathbf{d}$ and $\mathbf{2 a}$ is thermodynamically favorable. The activation energy of the 1,3-dipolar cycloaddition of diazo compounds 1 to thiobenzophenone (2a, $\left.\Delta G_{1}{ }^{\#}\right)$ is significantly larger than the decomposition energy of 1,3,4-thiadiazolines 6 $\left(\Delta G_{2}{ }^{\#}\right)$. In line with these data, the first step of the process (cycloaddition) must be a rate-determining step, and therefore, the larger value of $\Delta G_{1}{ }^{\#}$ corresponds to the slower formation of thiocarbonyl ylide 7 .

IRC scans have demonstrated that diazo compounds $\mathbf{1}$ and thiobenzophenone (2a) are smoothly converted to thiadiazolines $\mathbf{6}$ through the single minimum energy transition state, $\mathrm{TS}^{1}$. This observation implies that the cycloaddition reaction proceeds via a concerted mechanism. In the case of an alternative, stepwise

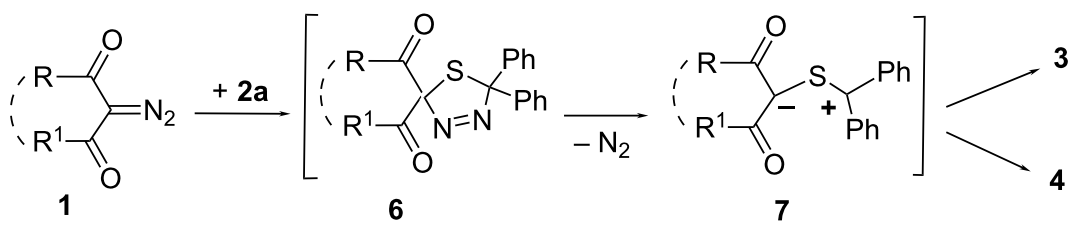

Scheme 2: General scheme for reactions of DDC 1 with thiobenzophenone (2a)

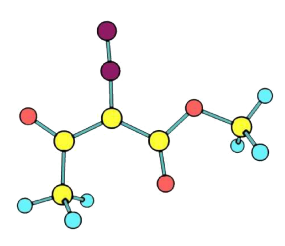

$1 \mathbf{a}$

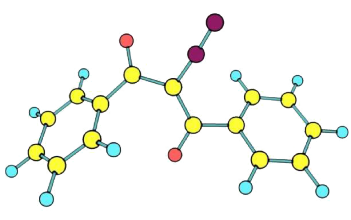

$1 \mathbf{b}$

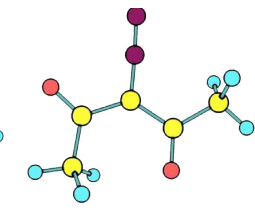

$1 \mathrm{c}$

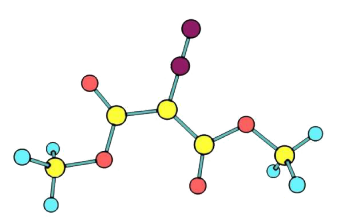

$1 d$

Figure 2: Optimized structures of the lowest energy Z,E-conformers of diazo compounds 1a-d. 
Table 1: Calculated relative energies (in $\mathrm{kcal} \cdot \mathrm{mol}^{-1}$ ) and relative reaction rates for cycloaddition reactions of diazo compounds $1 \mathrm{a}-\mathrm{g}$ with thiobenzophenone 2a using the PBE1PBE/6-31G*//PCM (benzene) method. The energies calculated via B3LYP/6-31G*//PCM (benzene) are given in parenthesis.

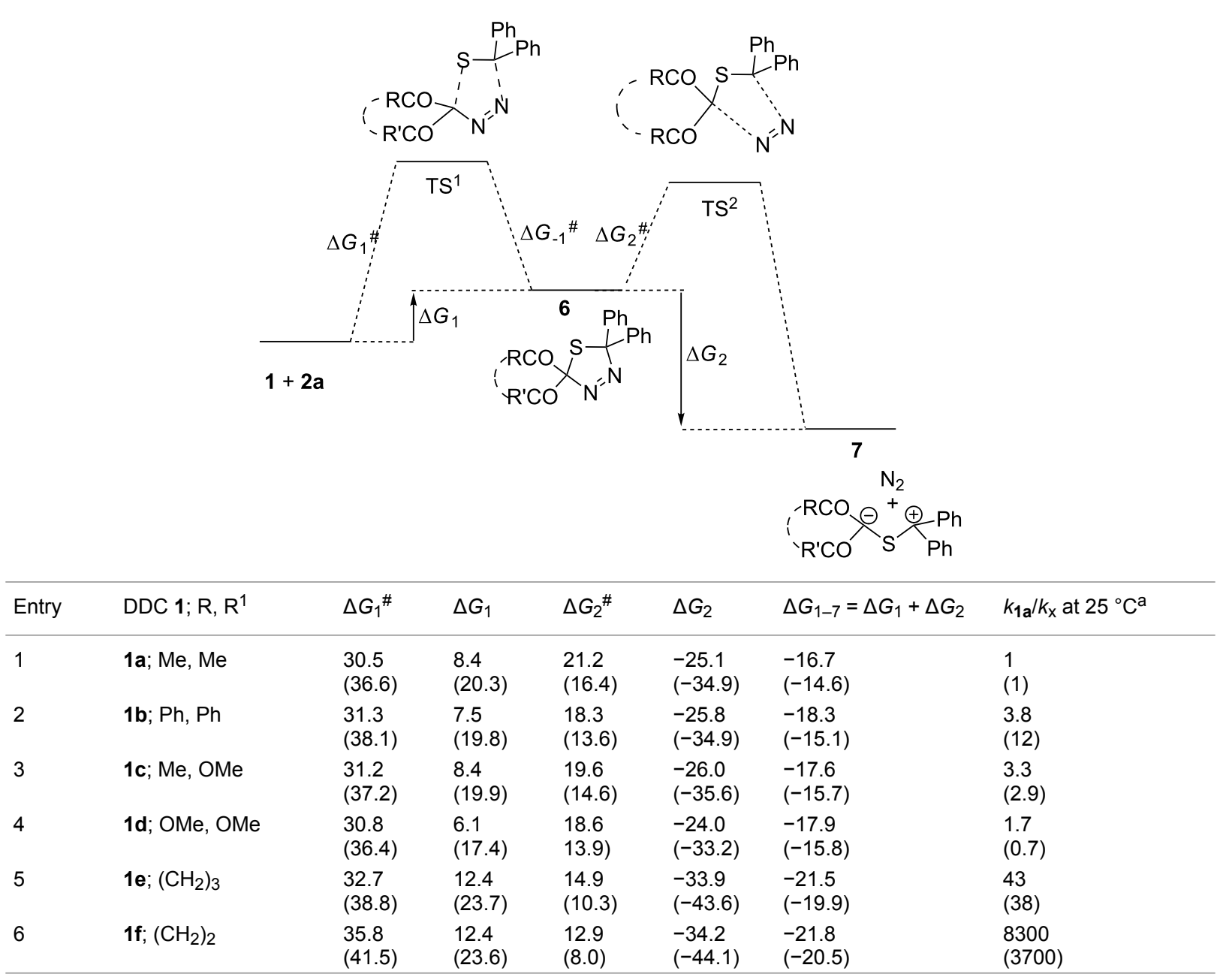

aThe details of $k_{1} \mathrm{a} / k_{\mathrm{x}}$ calculations are given in the Computational Details section.

mechanism, one would expect the appearance of at least two transition states [29], which was not observed. In addition, the cyclic geometry of the $\mathrm{TS}^{1}$ also confirms the concerted cycloaddition process.

The activation energies of cycloadditions of DDC $\mathbf{1}$ with thiobenzophenone (2a, Table 1) are in good agreement with the reported experimental results $[14,15,19]$. Thus, the smallest value of $\Delta G_{1}^{\#}\left(30.5 \mathrm{kcal} \cdot \mathrm{mol}^{-1}\right.$, PBE1PBE, Table 1, entry 1) corresponds to the reaction of the most reactive diazoacetylacetone (1a) with thioketone $\mathbf{2 a}$, which was completed at room temperature over several days (and in good yields of oxathiole 3a of up to $80 \%$ ). The intermediate values of $\Delta G_{1} \#$ (30.8-31.3 kcal $\cdot \mathrm{mol}^{-1}$, PBE1PBE, Table 1, entries 2-4) are related to diazodicarbonyl compounds $\mathbf{1} \mathbf{b}-\mathbf{d}$ with reaction times of 1-3 months. The carbocyclic diazodiketone $1 \mathrm{e}$ with
$\Delta G_{1}{ }^{\#}=32.7 \mathrm{kcal} \cdot \mathrm{mol}^{-1}(\mathrm{PBE} 1 \mathrm{PBE}$, Table 1, entry 5) does not react at room temperature with 2 a (Scheme 1), while diazocyclopentanedione $\mathbf{1 f}$ with the largest value of $\Delta G_{1}{ }^{\#}=35.8 \mathrm{kcal} \cdot \mathrm{mol}^{-1}$ (PBE1PBE, Table 1, entry 6) was unreactive under all conditions.

The calculated values of the relative reaction rates (Table 1, last right column) also correlate well with the above considered experimental data. The acyclic DDC 1b-d have much slower reaction rates as compared to diazoacetylacetone $\mathbf{1 a}$, whereas the cycloaddition of carbocyclic diazodiketones 1e,f with $\mathbf{2 a}$ occurs even at elevated temperatures at an insignificant rate. Apparently, these differences result from the fixed cyclic structure Z,Z-conformation of DDCs 1e,f, which is unfavorable for the 1,3-dipolar cycloaddition by stereochemical and/or the energetic parameters of the process. 


\section{Reactions with 2,2,4,4-tetramethyl-3-thioxo- cyclobutanone $\mathbf{( 2 b )}$}

The mechanism of the 1,3-dipolar cycloaddition of diazo compounds $\mathbf{1}$ with aliphatic thioketone $\mathbf{2} \mathbf{b}$ was assumed to be similar to their reaction with aromatic thione 2a (Scheme 2). Thus, the reaction of $\mathbf{1}$ with the $\mathrm{C}=\mathrm{S}$ bond of thioketone $\mathbf{2 b}$ (step 1) is followed by the decomposition of the intermediate 1,3,4-thiadiazoline 6' (step 2) giving rise to thiocarbonyl ylide 7'. The latter undergoes competitive 1,5- or 1,3-electrocylizations (step 3).
Considering that thiadiazolines with bulky substituents are rather stable compounds (in particular, those derived from thioketone $\mathbf{2 b}[18,30]$ ), it seems difficult to predict in advance whether step 1 or step 2 determines the reaction rate. In this respect, the relative energy of the stationary points on the potential energy surface for cycloaddition of diazo compounds 1a-f with cycloaliphatic thioketone $\mathbf{2 b}$ were calculated (Table 2). All calculations refer to the gas phase, since all reactions of DDC 1 with the thioxocyclobutanedione $\mathbf{2 b}$ were carried out under solvent-free conditions [20].

Table 2: Calculated relative energies for the 1,3-dipolar cycloadditions of diazodicarbonyl compounds $\mathbf{1 a - f}$ with thioketone $\mathbf{2 b}$ on the potential energy surface (in $\mathrm{kcal} \cdot \mathrm{mol}^{-1}$ ).

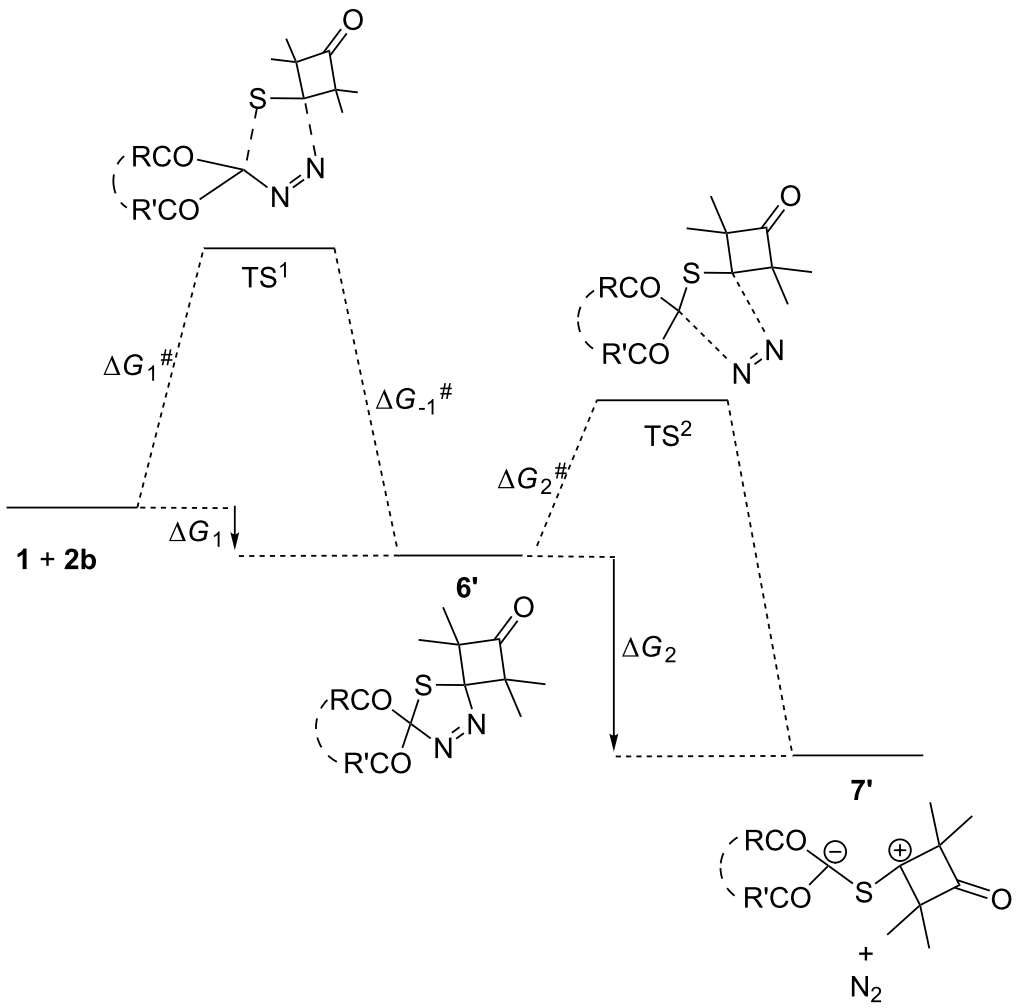

\begin{tabular}{|c|c|c|c|c|c|c|c|}
\hline Entry & Diazocompound & $\Delta G_{1}^{\#}$ & $\Delta G_{1}$ & $\Delta G_{2}^{\#}$ & $\Delta G_{2}$ & $\Delta G_{1-7^{\prime}}=\Delta G_{1}+\Delta G_{2}$ & $k_{1 a} / k_{x}$ at $25^{\circ} \mathrm{C}^{a}$ \\
\hline 1 & 1a; Me, Me & $\begin{array}{l}30.7 \\
(36.2)\end{array}$ & $\begin{array}{l}0.6 \\
(12.9)\end{array}$ & $\begin{array}{l}26.0 \\
(20.5)\end{array}$ & $\begin{array}{l}-12.8 \\
(-22.6)\end{array}$ & $\begin{array}{l}-12.2 \\
(-9.7)\end{array}$ & $\begin{array}{l}1 \\
(1)\end{array}$ \\
\hline 2 & 1b; Ph, Ph & $\begin{array}{l}29.3 \\
(34.7)\end{array}$ & $\begin{array}{l}-4.2 \\
(8.1)\end{array}$ & $\begin{array}{l}25.8 \\
(20.7)\end{array}$ & $\begin{array}{l}-8.3 \\
(-17.7)\end{array}$ & $\begin{array}{l}-12.5 \\
(-9.6)\end{array}$ & $\begin{array}{l}0.1 \\
(0.08)\end{array}$ \\
\hline 3 & 1c; Me, OMe & $\begin{array}{l}31.2 \\
(36.8)\end{array}$ & $\begin{array}{l}2.1 \\
(13.9)\end{array}$ & $\begin{array}{l}22.6 \\
(17.3)\end{array}$ & $\begin{array}{l}-14.8 \\
(-24.4)\end{array}$ & $\begin{array}{l}-12.7 \\
(-10.5)\end{array}$ & $\begin{array}{l}2.3 \\
(2.5)\end{array}$ \\
\hline 4 & 1d; OMe, OMe & $\begin{array}{l}30.8 \\
(36.2)\end{array}$ & $\begin{array}{l}-1.1 \\
(10.5)\end{array}$ & $\begin{array}{l}23.6 \\
(18.4)\end{array}$ & $\begin{array}{l}-13.3 \\
(-22.7)\end{array}$ & $\begin{array}{l}-14.4 \\
(-12.2)\end{array}$ & $\begin{array}{l}1.4 \\
(0.9)\end{array}$ \\
\hline 5 & $1 e ;\left(\mathrm{CH}_{2}\right)_{3}$ & $\begin{array}{l}31.9 \\
(37.3)\end{array}$ & $\begin{array}{l}5.6 \\
(17.5)\end{array}$ & $\begin{array}{l}16.0 \\
(10.7)\end{array}$ & $\begin{array}{l}-22.9 \\
(-33.2)\end{array}$ & $\begin{array}{l}-17.3 \\
(-15.7)\end{array}$ & $\begin{array}{l}7.7 \\
(6.2)\end{array}$ \\
\hline 6 & 1f; $\left(\mathrm{CH}_{2}\right)_{2}$ & $\begin{array}{l}33.8 \\
(39.4)\end{array}$ & $\begin{array}{l}4.6 \\
(15.9)\end{array}$ & $\begin{array}{l}14.8 \\
(10.0)\end{array}$ & $\begin{array}{l}-24.2 \\
(-33.6)\end{array}$ & $\begin{array}{l}-19.6 \\
(-17.7)\end{array}$ & $\begin{array}{l}210 \\
(210)\end{array}$ \\
\hline
\end{tabular}

aThe details of $k_{1} \mathrm{a} / k_{\mathrm{x}}$ calculations are given in the Computational Details section. 
The driving force behind the DDC 1a-d reactions with thiocyclobutanedione $\mathbf{2 b}$, similar to thiobenzophenone (2a), are the significant negative values of the Gibbs free energy change of the 1,3,4-thiodiazoline 6' decomposition process to produce thiocarbonyl ylide 7' $\left(\Delta G_{2}\right)$. This results in the overall negative total value of Gibbs free energy calculated according to the equation: $\Delta G_{1-7},=\Delta G_{1}+\Delta G_{2}$.

The lower relative energy of the 1,3,4-thiadiazoline 6' decomposition products, as well as the smaller activation energy of the second step of the process $\left(\Delta G_{2}{ }^{\#}<\Delta G_{1}{ }^{\#}\right)$, result in the formation of thiocarbonyl ylide $7^{\prime}$. Since the activation energy of the cycloaddition step is larger than that of step 2 (the formation of $\mathrm{C}=\mathrm{S}$ ylide), it is evident that step 1 determines the reaction rate. Furthermore, based on the character of the minimum energy transition states $\mathrm{TS}^{1}$, and by analogy with reactions of thiobenzophenone (2a), it is plausible to suggest that cycloadditions of DDC $\mathbf{1}$ with $\mathbf{2 b}$ also proceed via a concerted pathway.

The calculated activation energy of DDC $\mathbf{1 a}-\mathbf{f}$ cycloadditions with thioketone $\mathbf{2 b}$ correlate well with the experimental results, where the lowest values of $\Delta G_{1}{ }^{\#}\left(30.7-31.2 \mathrm{kcal} \cdot \mathrm{mol}^{-1}\right.$; Table 2) are related to the most reactive of acyclic diazoadicarbonyl compounds 1a-d [20] in this process. Carbocyclic diazodiketone 1e with $\Delta G_{1}{ }^{\#}=31.9 \mathrm{kcal} \cdot \mathrm{mol}^{-1}$ (PBE1PBE, Table 2, entry 5 ), in a similar way as the reaction with thiobenzophenone (2a), would not easily react at room temperature with thioketone $\mathbf{2 b}$. The largest $\Delta G_{1}{ }^{\#}=33.8 \mathrm{kcal} \cdot \mathrm{mol}^{-1}$ (PBE1PBE, Table 2, entry 6) corresponds to the lowest reaction rate of the carbocyclic diazodiketone 1f, which produces neither related oxathiole 3'f nor any other product in this reaction [20]. Apparently, the predicted reaction pathway stems from the unfavorable 1,3-cycloaddition process of the $Z, Z$ locked conformation of carbocyclic diazodiketones $\mathbf{1 e , f}$.

\section{Transformations of thiocarbonyl ylides 7 and 7'}

In order to evaluate the correlation between the structure of thiocarbonyl ylides 7, $\mathbf{7}^{\prime}$ and the direction of their electrocyclizations (1,5- or 1,3-dipolar electrocyclization), the relative energies of the stationary points on the potential energy surface of both reaction pathways were calculated (Table 3 ) for the electrocyclizations of transient species 7 and $\mathbf{7}^{\prime}$.

The negative values of the Gibbs free energy change for 1,5and 1,3-electrocyclizations of the ylides 7 and $7^{\prime}\left(\Delta G_{1}\right.$ and $\Delta G_{2}$, respectively), indicates that the reaction is thermodynamically allowed in both directions. However, based on the relative

Table 3: Calculated relative energies for competitive 1,5- and 1,3-electrocyclizations of thiocarbonyl ylides 7 and 7 ' on the potential energy surface (in $\left.\mathrm{kcal} \cdot \mathrm{mol}^{-1}\right){ }^{\mathrm{a}}$

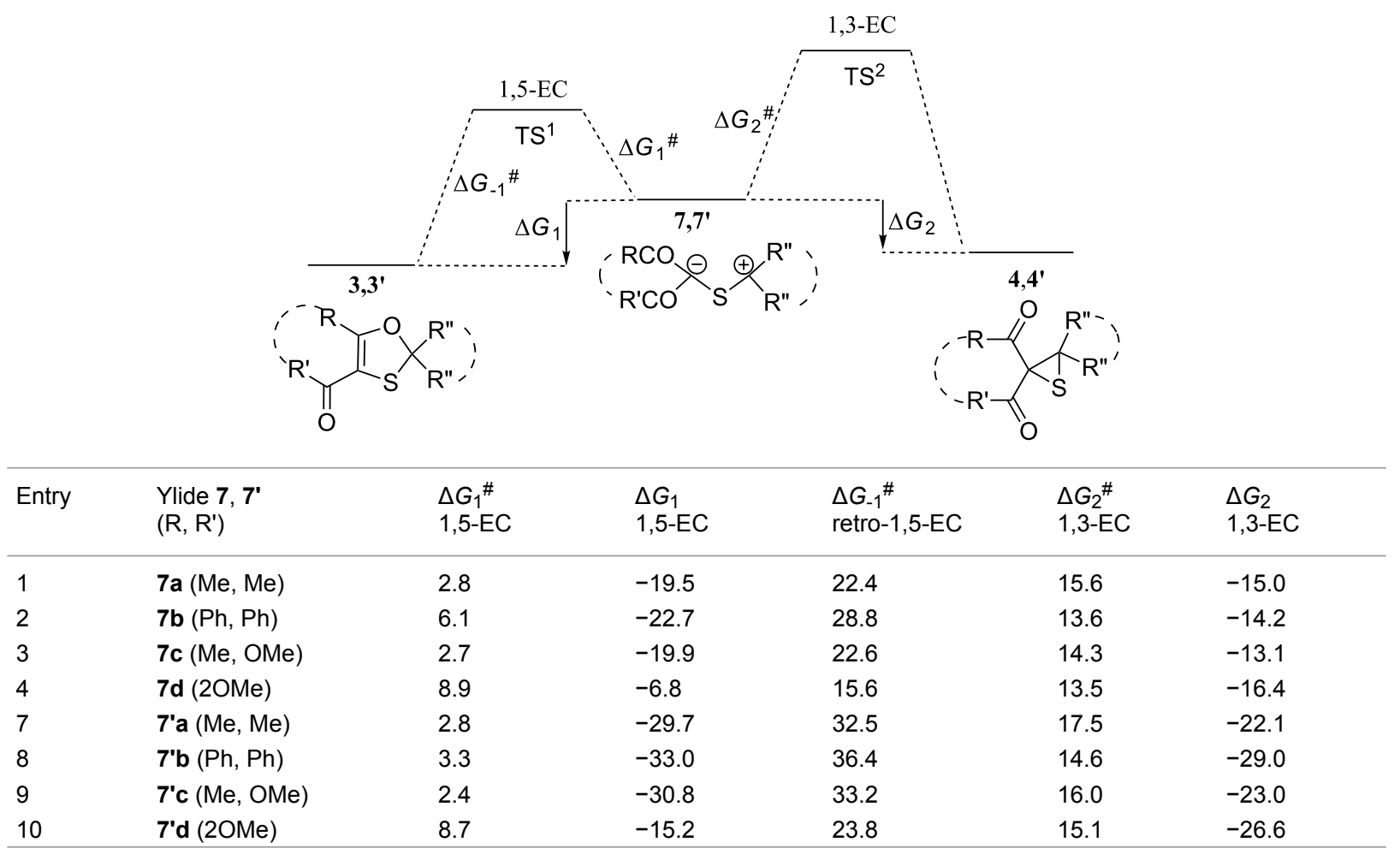

aPBE1PBE functional was solely used for calculations. 
values of $\Delta G_{1}$ and $\Delta G_{2}$ for thiocarbonyl ylides $7 \mathbf{a}-\mathbf{c}$ and $\mathbf{7} \mathbf{a}-\mathbf{c}$ (Table 3, entries 1-3 and 7-9), the 1,5-electrocyclization leading to 1,3-oxathioles $\mathbf{3} \mathbf{a}-\mathbf{c}$ and $\mathbf{3} \mathbf{a}-\mathbf{c}$ is a thermodynamically more favorable process. However, in the case of the diazomalonate derivatives $\mathbf{7 d}$ and $\mathbf{7}$ 'd (Table 3, entries 4 and $10)$, the 1,3-electrocyclization process is thermodynamically preferable, resulting in the formation of thiiranes $4 \mathbf{d}$ and 4 'd. At the same time, from the kinetic point of view, the 1,5-electrocyclization process is more efficient because the activation energy for the 1,5 -electrocyclization $\left(\Delta G_{1}{ }^{\#}\right)$ is usually smaller than for the 1,3-electrocyclization $\left(\Delta G_{2}{ }^{\#}\right)$.

The obtained computation results are in good agreement with the experimental observations, which demonstrate that at $20^{\circ} \mathrm{C}$, due to a kinetic reaction control, 1,3-oxathioles $\mathbf{3}$ and $\mathbf{3}$ ' are in most cases the major products of the multistep reactions of diazo compounds 1 with thioketones 2 [14,15,19,20]. At the same time, at elevated temperatures $\left(80^{\circ} \mathrm{C}\right)$, where the reaction is thermodynamically controlled, the lowest energy reaction products are expected to be formed. Indeed, the reaction of diazo compounds $1 \mathbf{a}-\mathbf{c}$ with thioketones $\mathbf{2 a}$ and $\mathbf{2 b}$ results in the formation of oxathioles $\mathbf{3 a}-\mathbf{c}$ and $\mathbf{3}$ 'a-c, while with diazomalonate 1d the thiiranes 4d, 4'd and alkenes 5d, 5'd, the desulfurization products of thiiranes are the principal reaction products [20].

To understand the mechanism of alkene 5'd formation and the effect of temperature on this process, the relative energy of the stationary points on the potential energy surface for the transformation of ylide 7'd into 1,3-oxathiole 3'd (path a), and further to alkene 5'd (path b) via the intermediate thiirane 4'd, were calculated (Scheme 3).
It was assumed that the mechanism includes the following steps [12,31,32]: 1,5-electrocyclization of $\mathrm{C}=\mathrm{S}$ ylide 7'd (step a), 1,3electrocyclization of $\mathrm{C}=\mathrm{S}$ ylide $\mathbf{7}$ ' $\mathbf{d}$ (step $\mathrm{b}$ ), disproportionation of the thiirane 4'd dimer into thiirane S-sulfide 8' $\mathbf{d}$ and alkene 5'd (step c). The subsequent decomposition of thiirane-Ssulfide 8'd (step d) and the tetramerization of the extruded $\mathrm{S}_{2}$ molecule results in the formation of the most thermodynamically stable, rhombic modification of sulfur $\mathrm{S}_{8}$ (step e). The obtained computational results for this process are summarized in Table 4 (the data for the Gibbs free energy change for the tetramerization of the $S_{2}$ molecule into $S_{8}$ were taken from the literature [33]).

According to the calculations, the Gibbs free energy changes for the 1,5-electrocyclization of ylide 7' $\mathbf{d}$ to 1,3 -oxathiole $\mathbf{3}$ ' $\mathbf{d}$ ( $\left.\Delta G_{1}\right)$, and/or 1,3-electrocyclization of ylide 7'd to thiirane 4'd, $\Delta G_{\mathrm{b}}$, are equal to -15.2 and $-26.6 \mathrm{kcal} \cdot \mathrm{mol}^{-1}$, respectively, whereas the relevant values of the activation energy for the steps a $\left(\Delta G_{\mathrm{a}}{ }^{\#}\right)$ and $\mathrm{b}\left(\Delta G_{\mathrm{b}}{ }^{\#}\right)$ are equal to 8.7 and $15.1 \mathrm{kcal} \cdot \mathrm{mol}^{-1}$, respectively. As a result, the thiirane derivative 4' $\mathbf{d}$ is thermodynamically more stable than 1,3-oxathiole 3'd, and therefore, 4'd and the corresponding alkene 5'd resulting from desulfurization are the main products formed at elevated temperatures when the reaction is thermodynamically controlled.

At the same time, the lower activation barrier of 1,5-electrocyclization $\left(\Delta G_{\mathrm{a}}{ }^{\#}=8.7 \mathrm{kcal} \cdot \mathrm{mol}^{-1}\right)$ as compared to 1,3 -electrocyclization $\left(\Delta G_{\mathrm{b}}{ }^{\#}=15.1 \mathrm{kcal} \cdot \mathrm{mol}^{-1}\right)$ results in the dominance of oxathiole $\mathbf{3}$ 'd, which is preferentially formed in the reaction mixture at room temperature when the reaction is kinetically controlled. Due to the relatively low activation barrier of the

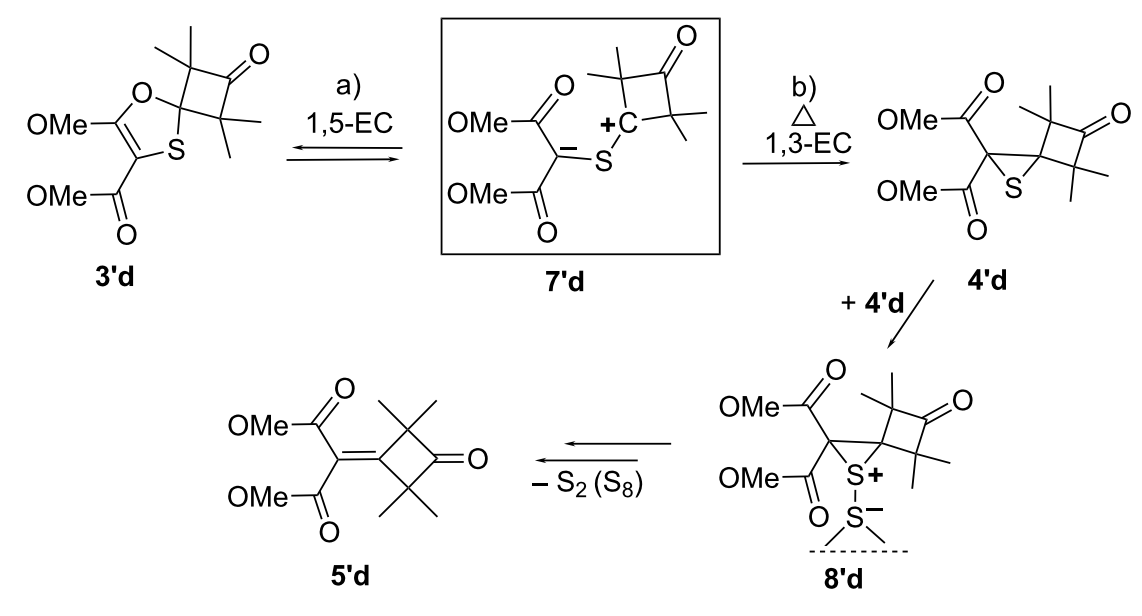

Scheme 3: Reactions of the intermediate thiocarbonyl ylide 7'd via competative 1,5-EC (a) or 1,3-EC (b) followed by desulfurization of thiirane 4'd into alkene $\mathbf{5}^{\prime} \mathbf{d}$. 
Table 4: Computed relative energies for conversion of thiocarbonyl ylide $\mathbf{7}$ 'd into 1,3-oxathiole 3'd and alkene $\mathbf{5}$ 'd on the potential energy surface (in $\left.\mathrm{kcal} \cdot \mathrm{mol}^{-1}\right)$.
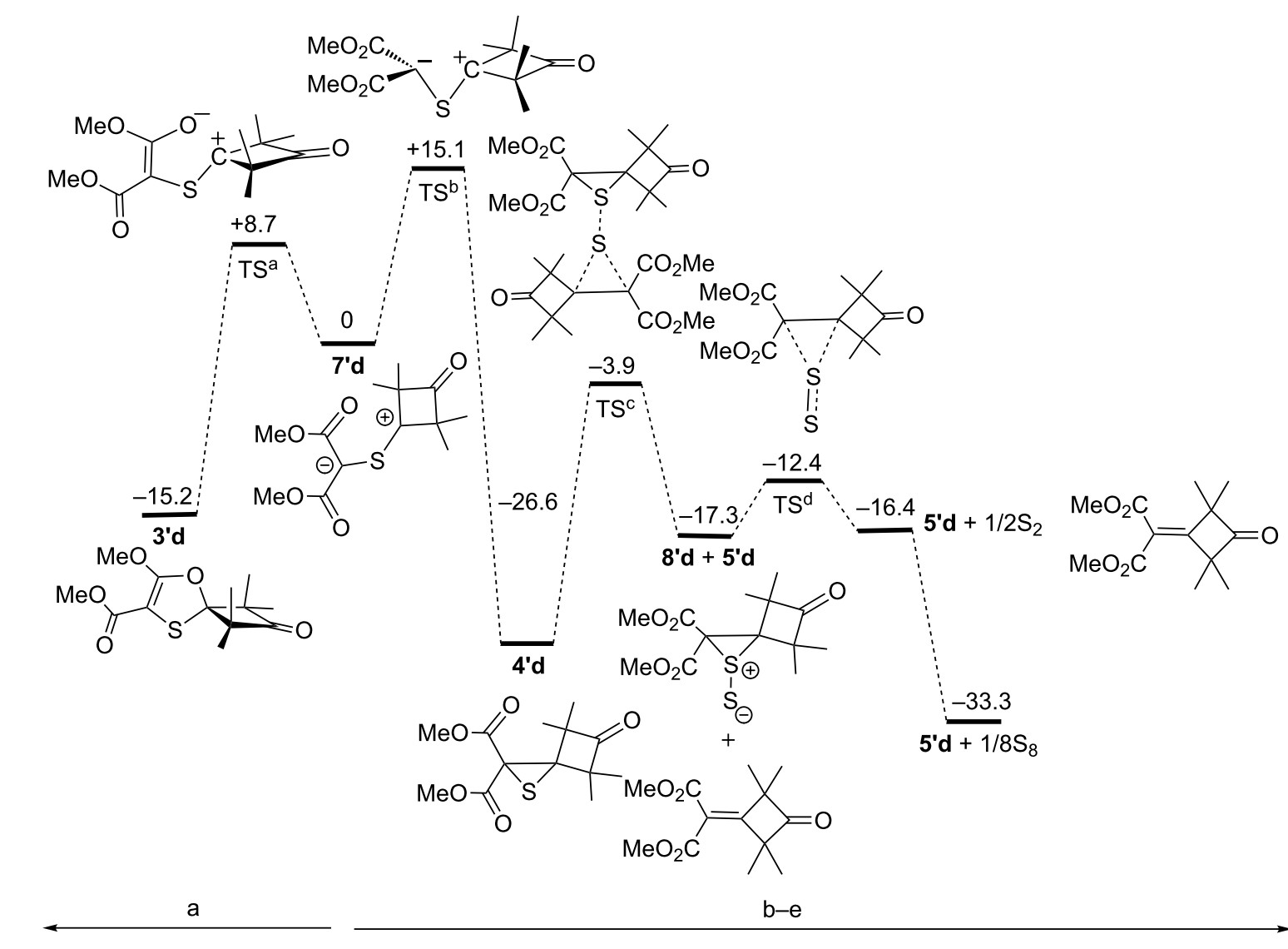

\begin{tabular}{|c|c|c|c|c|c|}
\hline Step & $a$ & $b$ & C & $d$ & $\mathrm{e}$ \\
\hline & 7'd $\rightarrow$ 3'd & 7'e $\rightarrow$ 4'd & $4 ' d \rightarrow 8^{\prime} d+5$ 'd & $8^{\prime} d \rightarrow 5^{\prime} d+S_{2}$ & $\mathrm{~S}_{\mathbf{2}} \rightarrow \mathrm{S}_{\mathbf{8}}$ \\
\hline$\Delta G_{i}$ & -15.2 & -26.6 & 9.3 & 0.9 & -16.9 \\
\hline$\Delta G_{i}^{\#}$ & 8.7 & 15.1 & 22.7 & 4.9 & - \\
\hline$\Delta G_{-i}^{\#}$ & 23.8 & 41.7 & 13.4 & 4.0 & - \\
\hline
\end{tabular}

1,3-oxathiole 3'd ring opening back into ylide 7'd $\left(\Delta G_{\text {-a }}{ }^{\#}=23.8 \mathrm{kcal} \cdot \mathrm{mol}^{-1}\right)$, the thermodynamically more stable alkene 5' $\mathbf{d}$ is accumulated in the reaction mixture with time even at room temperature.

As for a disproportionation mechanism of two thiirane molecules 4'd (step c) into thiirane S-sulfide 8'd and alkene 5'd, followed by the decomposition of the transient thiirane S-sulfide 8'd (step d), it is most likely that it follows the pathway proposed for the spontaneous desulfurization of matrix-isolated oxathiiranes [32].

\section{Conclusion}

The quantum-chemical calculations show that the initial step of the 1,3-dipolar cycloaddition of acyclic 2-diazo-1,3-dicarbonyl compounds with the $\mathrm{C}=\mathrm{S}$ bonds of "superdipolarophilic" thioketones proceeds with $Z, E$-conformers of DDC via a concerted mechanism and adequately agree with experimental data. At room temperature, the 1,5-electrocyclization of the intermediate ylides into the 1,3-oxathiole derivatives is a thermodynamically and kinetically favorable process for DDC with at least one electron-withdrawing acyl group. At higher temperatures, however, due to the entropy contribution, the dominating process becomes the 1,3-cyclization followed by desulfurization of the obtained thiiranes, which leads to the corresponding alkenes. In the case of the $\mathrm{C}=\mathrm{S}$ ylides bearing two alkoxycarbonyl groups, the latter pathway clearly dominates. The reversibility of the 1,5-electrocyclization of the transient $\mathrm{C}=\mathrm{S}$ ylide, generated from diazomalonate $\mathbf{1 d}$ and thione $\mathbf{2 b}$, is attributed to a relatively low activation barrier $\left(\Delta G^{\#}=23.8 \mathrm{kcal} \cdot \mathrm{mol}^{-1}\right)$ for the ring opening of the 1,3 oxathiole back to thiocarbonyl ylide. Carbocyclic diazodike- 
tones are practically unreactive at room temperature due to the locked Z,Z-configuration of the diazo molecule, which is unfavorable for initiating the cycloaddition step.

\section{Computational Details}

The geometry optimization of reagents, intermediates, products, and transition states, and the calculations of the molecular orbital energies, vibrational frequencies, and ground-state IRC scans, were all performed at the B3LYP and PBE1PBE theory level employing the 6-31G(d) basis set using the GAUSSIAN 09 program package [34]. The calculated energies for the B3LYP and PBE1PBE functionals were found to be consistent. The thermochemical parameters were calculated for $298.15 \mathrm{~K}$ and all are given in $\mathrm{kcal} \cdot \mathrm{mol}^{-1}$. For the reactions of diazo compounds 1a-f with thiobenzophenone (2a), the solvent was simulated using the polarizable continuum model (PCM) [22]. All calculations for the reactions of diazo compounds $\mathbf{1 a}, \mathbf{c}, \mathbf{d}$ with thiocyclobutanedione $\mathbf{2 b}$ were performed in the gas phase. To characterize the nature of the stationary points, the corresponding vibrational frequencies were calculated. The optimized structure of the reagents, intermediates, and products was characterized by the absence of an imaginary frequency, and transition states containing only one imaginary frequency component. The calculated IRC paths demonstrated that the stationary points are effectively connected to each other. The relative reaction rates were estimated using the Arrenius equation (Equation 1), where $k$ is the reaction rate, $A$ is the prefactor, $\Delta G^{\#}$ is the activation energy, $R$ is the universal gas constant, and $T$ is the temperature.

$$
\begin{gathered}
k=A \cdot e^{\frac{-\Delta G^{\#}}{R T}} \\
k_{1 a} / k_{x}=e^{\frac{\Delta G_{1}^{\#}(x)-\Delta G_{1}^{\#}(a)}{R T}}
\end{gathered}
$$

The relative reaction rates of DDC $\mathbf{1 b}-\mathbf{f}$, as compared to the most reactive diazoacetylacetone 1a, were estimated by Equation 2 (Table 1 and Table 2).

\section{Supporting Information}

\section{Supporting Information File 1}

Details of computational studies: cartesian coordinates, computed geometries of compounds, transition states, and computed total energies.

[http://www.beilstein-journals.org/bjoc/content/ supplementary/1860-5397-11-57-S1.pdf]

\section{Acknowledgements}

A. V. I. thanks the Saint Petersburg State University for financial support of his stay at the University of Łódź with Prof. G. Mloston (order 1831/1; 02.06.2011). A. S. M. acknowledges the Saint Petersburg State University for financial support in the form of a postdoctoral fellowship (No. 12.50.1562.2013). G. M. acknowledges support by the National Science Center (PLCracow) within the Grant Maestro-3 (Dec-2012/06/A/ST5/ 00219). The calculations were performed with the assistance of the Saint Petersburg State University Computer Center and the Chemistry Department of Saint Petersburg State University.

\section{References}

1. Huisgen, R. Angew. Chem. 1955, 67, 439-463. doi:10.1002/ange.19550671703

2. Padwa, A.; Pearson, W. H., Eds. Synthetic Applications of 1,3-Dipolar Cycloaddition Chemistry Toward Heterocycles and Natural Products; Wiley: New York, 2002; Vol. 59.

3. Padwa, A., Ed. 1,3-Dipolar Cycloaddition Chemistry; Wiley: New York, 1984; Vol. 1 and 2.

4. Zollinger, H. Diazo Chemistry II; VCH: Weinheim, 1995.

5. Korobizina, I.; Rodina, L. L. Aliphatische Diazoverbindungen. In Methodicum Chimicum; Korte, F., Ed.; Georg Thieme Verlag: Stuttgart, 1974; Vol. 6, pp 260-307.

6. Huisgen, R.; Grashey, R.; Sayer, G. 1,3-Dipolar Cycloaddition. In The Chemistry of Alkenes; Patai, S., Ed.; Khimia: Leningrad, 1969; pp 481-534.

7. Korobitsyna, K.; Bulusheva, V. V.; Rodina, L. L. Chem. Heterocycl. Compd. 1978, 14, 471-486. doi:10.1007/BF00673325

8. Huisgen, R.; Verderol, M. P. B.; Gieren, A.; Lamm, V. Angew. Chem., Int. Ed. Engl. 1981, 20, 694-695. doi:10.1002/anie.198106941

9. Huisgen, R.; Reissig, H.-U.; Huber, H. J. Am. Chem. Soc. 1979, 101, 3647-3648. doi:10.1021/ja00507a035

10. Huisgen, R.; Reissig, H.-U. Angew. Chem., Int. Ed. Engl. 1979, 18, 330-331. doi:10.1002/anie.197903301

11. Kelmendi, B.; Mlostoń, G.; Heimgartner, H. Heterocycles 2000, 52, 475-482. doi:10.3987/COM-99-S35

12. Mlostoń, G.; Heimgartner, H. Helv. Chim. Acta 1996, 79, 1785-1792. doi:10.1002/hlca.19960790704

13. Huisgen, R.; Langhals, E. Tetrahedron Lett. 1989, 30, 5369-5372. doi:10.1016/S0040-4039(01)93789-X

14. Nikolaev, V. A.; Ivanov, A. V.; Shakhmin, A. A.; Schulze, B.; Rodina, L. L. Russ. J. Org. Chem. 2011, 47, 1911-1913.

15. Mlostoń, G.; Pipiak, P.; Linden, A.; Heimgartner, H. Helv. Chim. Acta 2015, 98, 190-200. doi:10.1002/hlca.201400334

16. Mlostoń, G.; Heimgartner, H. Curr. Org. Chem. 2011, 15, 675-693. doi:10.2174/138527211794518961

17. Mlostoń, G.; Heimgartner, H. Thiocarbonyl Ylides. In The Chemistry of Heterocyclic Compounds; Padwa, A.; Pearson, W. H., Eds.; Wiley: New York, 2002; Vol. 59, pp 315-360.

18. Mlostoń, G.; Heimgartner, H. Pol. J. Chem. 2000, 74, 1503-1530.

19. Nikolaev, V. A.; Ivanov, A. V.; Shakhmin, A. A.; Sieler, J.; Rodina, L. L. Tetrahedron Lett. 2012, 53, 3095-3099. doi:10.1016/j.tetlet.2012.04.036 
20. Nikolaev, V. A.; Ivanov, V.; Rodina, L. L.; Mlostoń, G.

Beilstein J. Org. Chem. 2013, 9, 2751-2761. doi:10.3762/bjoc.9.309

21. Ivanov, A. V. Reactions of 2-diazo-1,3-dicarbonyl compounds with thioketones and acetylenic dipolarophiles: synthesis of S-, N-, and O-containing heterocycles. Ph.D. Thesis, Saint Petersburg State University, Saint Petersburg, Russia, 2014.

22. Tomasi, J.; Mennucci, B.; Cammi, R. Chem. Rev. 2005, 105, 2999-3094. doi:10.1021/cr9904009

23. Houk, K. N.; Sims, J.; Watts, C. R.; Luskus, L. J. J. Am. Chem. Soc. 1973, 95, 7301-7315. doi:10.1021/ja00803a018

24. Sustmann, R. Pure Appl. Chem. 1974, 40, 569-593. doi:10.1351/pac197440040569

25. Sustmann, R. Tetrahedron Lett. 1971, 12, 2717-2720. doi:10.1016/S0040-4039(01)96961-8

26. Lauer, W.; Krause, V.; Wengenroth, H.; Meier, H. Chem. Ber. 1988, 121, 465-469. doi:10.1002/cber.19881210313

27. Nikolaev, V. A.; Popik, V. V.; Korobitsyna, I. K. J. Org. Chem. USSR 1991, 27, 437-450.

28. Bogdanova, A.; Popik, V. V. J. Am. Chem. Soc. 2004, 126, 11293-11302. doi:10.1021/ja047824r

29. Firestone, R. A. J. Org. Chem. 1968, 33, 2285-2290. doi:10.1021/jo01270a023

30. Huisgen, R.; Langhals, E.; Polborn, K.; Karaghiosoff, K. Helv. Chim. Acta 2004, 87, 1426-1445. doi:10.1002/hlca.200490130

31. Mlostoń, G.; Heimgartner, H. Pol. J. Chem. 2002, 76, 551-555.

32. Reisenauer, H. P.; Mlostoń, G.; Romański, J.; Schreiner, P. R. Eur. J. Org. Chem. 2011, 6269-6275. doi:10.1002/ejoc.201100695

33. Holleman, A. F.; Wiberg, N. Inorganic Chemistry; Walter de Gruyter GmbH: Berlin, 2001; pp 505-509.

34. Gaussian 09, revision A.02; Gaussian, Inc.: Wallingford, CT, 2009.

\section{License and Terms}

This is an Open Access article under the terms of the Creative Commons Attribution License (http://creativecommons.org/licenses/by/2.0), which permits unrestricted use, distribution, and reproduction in any medium, provided the original work is properly cited.

The license is subject to the Beilstein Journal of Organic Chemistry terms and conditions:

(http://www.beilstein-journals.org/bjoc)

The definitive version of this article is the electronic one which can be found at: doi:10.3762/bjoc. 11.57 